\title{
THE EFFECT OF THE TRANSIT OF VENUS ON ACRIM'S TOTAL SOLAR IRRADIANCE MEASUREMENTS: IMPLICATIONS FOR TRANSIT STUDIES OF EXTRASOLAR PLANETS
}

\author{
G. SCHNEIDER \\ Steward Observatory, The University of Arizona, 933 North Cherry Avenue, Tucson, AZ 85721; \\ gschneider@as.arizona.edu \\ J. M. PASACHOFF \\ Williams College, Williamstown, MA 01267; jay.m.pasachoff@williams.edu \\ AND \\ RICHARD C. WILLSON \\ Columbia University, New York, NY 10027; rwillson@acrim.com \\ Received 2005 November 15; accepted 2005 December 8
}

\begin{abstract}
We have used the 2004 June 8 transit of Venus (ToV) as a surrogate to test observing methods, strategies, and techniques that are being contemplated for future space missions to detect and characterize extrasolar terrestrial planets (ETPs) as they transit their host stars, notably NASA's Kepler mission, planned for 2008. As an analog to "Kepler-like" photometric transit observations, we obtained (spatially unresolved) radiometric observations with the ACRIM 3 instrument on ACRIMSAT at a sampling cadence of $131 \mathrm{~s}$ to follow the effect of the ToV on the total solar irradiance (TSI). Contemporaneous high-resolution broadband imagery with NASA's TRACE spacecraft provided, directly, measures of the stellar (solar) astrophysical noise that can intrinsically limit such transit observations. During the Venus transit, which lasted $\sim 5.5 \mathrm{hr}$, the planet's angular diameter was approximately $1 / 32$ the solar diameter, thus covering $\sim 0.1 \%$ of the stellar surface. With our ACRIM 3 data, we measure temporal changes in TSI with a $1 \sigma$ per sample (unbinned) uncertainty of approximately $100 \mathrm{~mW} \mathrm{~m}^{-2}(0.007 \%)$. A diminution in TSI of $\sim 1.4 \mathrm{~W} \mathrm{~m}^{-2}$ ( $\sim 0.1 \%$, closely corresponding to the geometrically occulted area of the photosphere) was measured at mid-transit compared with a mean pre-/post-transit TSI of $\sim 1365.9 \mathrm{~W} \mathrm{~m}^{-2}$. The radiometric light curve is complex because of the parallactic motion of Venus induced by ACRIMSAT's near-polar orbit, but exhibits the characteristic signature of photospheric limb darkening. These observations serve as a surrogate for future photometric observations of ETPs, such as Kepler will deliver. Detailed analysis of the ToV, a rare event within our own solar system, with time-resolved radiometry augmented with high-resolution imagery, provides a useful analog for investigating the detectability and characterization of ETPs from observations that are anticipated in the near future.
\end{abstract}

Subject headings: planets and satellites: individual (Venus) — techniques: photometric

\section{INTRODUCTION}

Since the seminal detection and identification of the companion to $51 \mathrm{Peg}$ as a giant planet orbiting very close to its host star (Mayor \& Queloz 1995), the past decade has seen an explosion of detections of extrasolar "hot Jupiter"-type planets around solar-like stars. ${ }^{1}$ Direct detection of young $(<$ few tens of megayears) gravitationally bound companions of planetary mass (predicated on evolutionary cooling models) to nearby $(<$ few tens of parsecs) stars via high-contrast space-based coronagraphy (e.g., Schneider \& Silverstone 2003) and/or ground-based adaptive optics augmented imaging in the near-IR (e.g., Macintosh et al. 2001) is now technically feasible. Detection (and eventually characterization) of terrestrial planets around solar-like stars at an advanced stage of evolution and in their "habitable zones" remains a challenging, although yet unfulfilled, key goal in observational astronomy and astrophysics. Major space-based initiatives such as the Kepler (Borucki et al. 2003) and even more technically challenging Terrestrial Planet Finder (TPF; Beichman

\footnotetext{
${ }^{1}$ See the Extrasolar Planets Encyclopedia at http://vo.obspm.fr/exoplanetes/ encyclo/catalog-main.php and the California \& Carnegie Planet Search page at http://exoplanets.org/.
}

1998) missions are under way and development and, one hopes, will rise to this challenge.

Indirect detection of Jovian-mass extrasolar planets has been demonstrated by the transit method (e.g., HD 209458b, Charbonneau et al. 2000; TrES-1, Alonso et al. 2004). Fledgling steps have been taken toward exoplanet atmospheric identification and characterization by transit spectroscopy as well (e.g., HD 209458b with the detection of atmospheric sodium; Charbonneau et al. 2002) and conceptually may be extended, with a much higher degree of observational complexity, to ETPs yet to be found. The recent predicted transit of a known terrestrial planet in the habitable zone of a very nearby solar"like" star, i.e., the transit of Venus across the Sun on 2004 June 8 (Pasachoff et al. 2005), provided a unique and timely opportunity to validate the concepts, instrumentation, observational processes, and procedures that will be developed over the next decade to extend our vision of terrestrial planets to other solar systems. Seizing on that rare opportunity, we obtained spacebased solar irradiance (total irradiance power) measurements with the Active Cavity Radiometer Irradiance Monitor $^{2}$ (ACRIM) 3 instrument on ACRIMSAT to evaluate the radiometric (closely

\footnotetext{
${ }^{2}$ See current ACRIM projects at http://www.acrim.com/.
} 
analogous to broadband photometric) detectability of spatially unresolved ETP transits of their host stars using the ToV as a "nearby" analog. ${ }^{3}$ With contemporaneously obtained highresolution broadband imagery of the transit obtained with the Transition Region and Coronal Explorer (TRACE) ${ }^{4}$ spacecraft to evaluate the intrinsic stellar (i.e., solar) "astrophysical" noise, we compare transit light-curve-derived systemic results with our a priori knowledge of the characteristics of the Venus/ Sun system.

\section{OBSERVATIONS AND INSTRUMENTATION}

Both the ACRIMSAT and TRACE spacecraft provide unique "views" of the Sun from low Earth orbit (LEO), each offering instruments well suited to this investigation. The ACRIM 3 radiometer (Willson \& Mordvinov 2003) provides the total ( 0.2 $2.0 \mu \mathrm{m}$ ) solar irradiance (measured in watts per square meter received at $1 \mathrm{AU}$ ). The white-light (WL) channel of the TRACE imager (Strong et al. 1994) provides high $\left(\sim 00^{\prime \prime} 5\right)$ spatial resolution broadband $(0.1-1.0 \mu \mathrm{m})$ solar photospheric imagery. While TRACE observes the Sun continuously, ACRIMSAT's observations are interrupted for $\sim 30$ minutes by Earth occultation during each of its $\sim 100$ minute orbits.

\subsection{ACRIM 3 Radiometry}

ACRIM 3 was designed to provide accurate, highly precise, and traceable radiometry over decadal timescales, to detect changes in the total energy received from the Sun by the Earth (e.g., Fig. 1). ACRIM 3's long-term radiometric traceability (including self-calibration of sensor degradation) is $0.0003 \% \mathrm{yr}^{-1}$.

The basic TSI data obtained by ACRIM 3 are the average of $32 \mathrm{~s}$ of sampling (at a $1.024 \mathrm{~s}$ cadence) during each set of shutter open (observations) and closed (calibration) measurements. While standard data products are binned into daily means, ACRIM 3 also provides shorter timescale calibrated measures (a "shutter cycle" readout) with a $1 \sigma$ single-sample uncertainty of $\sim 0.1 \%$ and a precision of $\sim 0.01 \%$ (100 $\mu \mathrm{mag}$ in the extremely broad radiometric ACRIM 3 "passband"). The ACRIM 3 instrument delivers these measures once every $131.072 \mathrm{~s}$. The radiometric data we discuss in this paper were derived from these measures that were obtained during, and flanking, the 2004 June $8 \mathrm{ToV}$ from 01 to $16 \mathrm{hr}$ UT.

\subsection{TRACE Imagery}

Using the TRACE WL channel, which provides spectral sensitivity in the wavelength range from 0.12 to $0.96 \mu \mathrm{m}$, we obtained 98 image frames during the transit ingress from 05:05:53 to $05: 47: 51$ UT and 84 frames during egress from 10:52:31 to 11:23:15 UT. Because of the need for high interframe temporal cadence in the highest fidelity available data format, we read out only a $512 \times 512$ subarray of the $1024 \times 1024$ CCD detector due to both downlink bandwidth and memory (image buffer) limitations. Our observations were interleaved with those of other programs (using other TRACE filters and data formats); thus, the interframe temporal spacing was not uniform throughout our imaging sequences. During ingress we achieved a cadence of five frames per minute (uniformly spaced) across the limb contacts, but at a reduced rate of $\sim$ two frames per minute at other times.

\footnotetext{
${ }^{3}$ Symbiotic areas of scientific inquiry enabled through suggested observations of this rare event are summarized by the NASA Astrobiology Institute Astronomy Focus Group on the Transit of Venus at http://www.dtm.ciw.edu/seager/ NAIAFG/AFGVenus.html.

${ }^{4}$ See the TRACE Web site at http://trace.lmsal.com/.
}

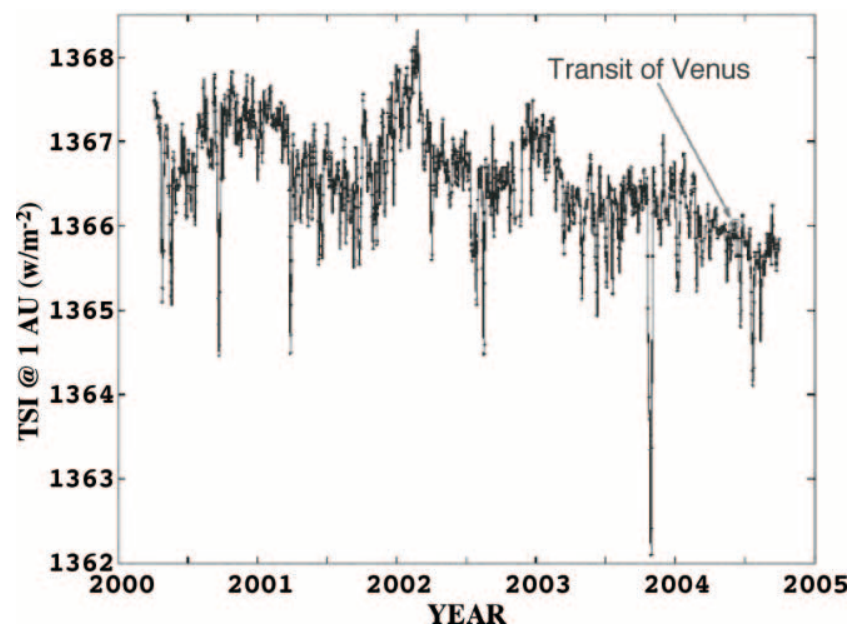

FIG. 1.-Daily mean TSI (660 shutter cycle readouts per point corrected to $1 \mathrm{AU}$ ) from ACRIM 3 during the interval 2000.2-2004.7. On 2004 June 8 (the ToV epoch) the TSI (formerly the "solar constant") was $1365.9878 \mathrm{~W} \mathrm{~m}^{-2}$, excluding measures during the transit itself $(05.0-11.5 \mathrm{UT})$. The long-term solar variability (on timescales of years) of $\sim 0.2 \%$ is representative of what is expected from solar-analog targets to be observed by the Kepler mission with multiple observations of early G-type stars during the course of its $4 \mathrm{yr}$ mission.

At egress we obtained images at third contact \pm 3 minutes at a uniformly spaced rate of seven frames per minute, but at a slower rate of $\sim$ one frame every $35 \mathrm{~s}$ when the leading edge of Venus was farther from the solar limb. The image data were processed as described by Schneider et al. (2004), where additional information on the TRACE WL channel for planetary transit imaging may be found.

The TRACE WL optical channel, so we discovered, suffers from an $\sim 1 \%$ intensity optical "ghosting." Normally, this is not of concern for TRACE's solar observation programs, but our interest rested, primarily, in the bottom $1 \%$ of the (12-bit) dynamic sampling range. With detailed characterization (including the analysis of identically acquired pre-transit imagery), we found the ghosting to be double, with different degrees of afocality and intensity, and an additional diffuse (not afocally specular) component. For each processed image frame, we built and subtracted models of the instrumentally scattered light to remove these artifacts before photometric measures were later made. ${ }^{5}$

During the transit, Venus's angular diameter was $58.22(12,104 \mathrm{~km}$ at $0.289 \mathrm{AU}$ ), diametrically spanning $116 T R A C E$ detector pixels. The TRACE WL spatial resolution is Nyquist-limited by its $0.5 \mathrm{pixel}^{-1}$ sampling of its CCD, not diffraction-limited by the $0.3 \mathrm{~m}$ diameter telescope. With a WL flux-weighted mean wavelength of $0.62 \mu \mathrm{m}$, Venus was resolved by $\sim 60$ band-integrated resolution elements.

\section{THE ACRIMSAT ORBIT}

ACRIMSAT is in a Sun-synchronous orbit, but unlike TRACE, it cannot observe the Sun continuously. ACRIMSAT's line of sight to the Sun is occulted by the Earth during each spacecraft orbit. As a result of its orbital geometry ACRIM 3 is actively measuring the TSI for about an hour orbit in each of its approximately 100 minute orbits. These periodic interruptions are not

\footnotetext{
5 A full description of the ghost removal process, along with representative post-processed image frames after additionally removing the radial intensity gradient induced by solar limb darkening, may be found at $h t t p: / / n i c m o s i s . a s . a r i z o n a$ .edu:8000/ECLIPSE_WEB/TRANSIT_04/TRACE/TOV_TRACE.html.
} 


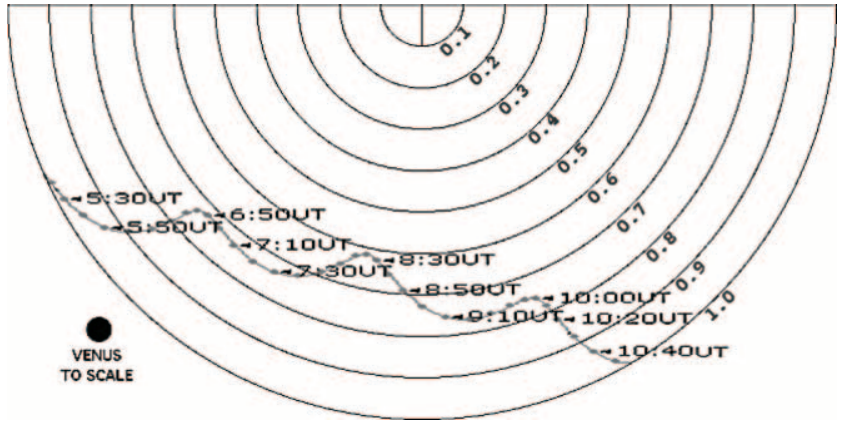

FIG. 2.-Apparent path of Venus crossing the face of the Sun as seen from ACRIMSAT. Points plotted in 10 minute intervals illustrate the nonlinear motion in the heliocentric frame. Radial distances (in solar radii) from the heliocenter are indicated in $0.1 R_{\odot}$ increments.

of concern to the primary mission for ACRIM-to monitor with higher precision long-term variations in the solar radiometric output. This does mean, however, that our ToV data set, which otherwise nominally provides samples once every $131.072 \mathrm{~s}$, "suffers" from intraorbit gaps due to visibility interruptions.

The line-of-sight solar visibility intervals and Venus transit circumstances, as seen by ACRIMSAT, were determined for time intervals spanning the ToV using a definitive (post priori) orbital ephemeris derived from a contemporaneous epochal satellite element set provided by the North American Aerospace Defense Command.

\subsection{ACRIMSAT's "View" of the Transit}

The apparent path of (the center of) Venus as seen from $A C R I M S A T$ with respect to the heliocenter as the planet crossed the face of the Sun is depicted in Figure 2. The planetary parallax (shifting of the line of sight to Venus) induced by the spacecraft orbit, projected onto the disk of the Sun, causes periodic spatial and temporal modulations in the location of Venus as it traverses the solar disk. The "vertical" amplitude variations (i.e., in the north-south direction in this "north up" illustration) result from the near-polar ACRIMSAT orbit. As Venus is of similar size as the Earth, and as ACRIMSAT is in a low Earth orbit, the vertical excursions are also comparable to (but a bit larger than) the diameter of Venus. The "horizontal" (east-west) component manifests itself in nonlinear spacings in the planetary position along its projected path in equal time intervals. This variation results from the ACRIMSAT orbit plane not being in the line-of-sight direction to the Sun. With TRACE the modulation is more closely sinusoidal, as its orbit plane is perpendicular to the Earth/Sun line, but deviates from a true sinusoid (linear in spacecraft orbital phase angle with time) because of the planet's orbital motion about the Sun.

\section{THE ACRIM 3 RADIOMETRIC "LIGHT CURVE"}

The disk of Venus, fully silhouetted in front of the Sun as viewed by ACRIMSAT during the ToV, occulted $\sim 0.1 \%$ of the total area of the photosphere, so a radiometric detection of the transit by ACRIM 3 was readily expected - and was obtained. The ACRIM 3 ToV data, provided and radiometrically calibrated by the ACRIM Experiments team (extending $5 \mathrm{hr}$ on the external sides of the transit ingress and egress), are shown in Figure 3 as the radiometric light curve. Gaps in the ACRIM 3 light curve primarily result from Earth occultations, although a small amount of data were irreparably lost elsewhere in the downlink path from the spacecraft. Unfortunately, the transit egress (the interval between contacts III and IV) was not visible from ACRIMSAT.

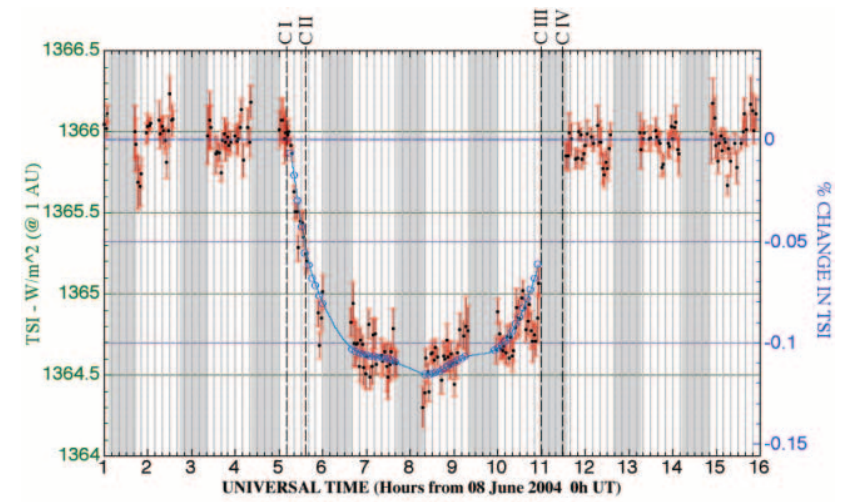

FIG. 3.-ACRIM 3 radiometric light curve of the 2004 June 8 ToV (in UT). Black points, and their associated red error bars, are the $131 \mathrm{~s}$ time-resolved ACRIM 3 TSI measures and their $1 \sigma$ measurement uncertainties. Blue circles are "expected" values from a geometric orbit and solar limb-darkening model ( $\$$ ) during the transit at 5 minute intervals while the Sun was visible to ACRIMSAT. The Sun, as seen from ACRIMSAT, was occulted by the Earth during the times indicated by the gray bars; thus, no data were available during those intervals. The vertical dashed lines labeled CI-CIV indicate the instants of geometric tangency of the Cytherian and solar disks based on the ACRIMSAT orbital ephemeris.

TSI observations were also obtained from the Total Irradiance Monitor (TIM) instrument on the Solar Radiation and Climate Experiment (SORCE) spacecraft. ${ }^{6,7}$ Coincidentally (and unfortunately), the phasing of the SORCE orbit with respect to its periods of Earth occultation during the ToV was very similar to ACRIMSAT's, and egress was also unobserved by TIM. As we use only the ACRIM 3 data in our analysis, we do not discuss the TIM data further. We also note that the Solar and Heliospheric Observatory spacecraft (in a halo orbit about the L1 Lagrangian point rather than LEO) was not in a zone from which the transit was visible; thus, transit observations from the Variability of Irradiance and Gravity Oscillations instrument were not possible.

\section{A MODEL LIGHT CURVE}

We tested the recoverability of the a priori known transit parameters (i.e., the geometrically derived transit depth and contact times, knowing $d_{\text {Venus }} / d_{\text {Sun }}$ and positions of Venus with respect to the heliocenter as a functions of time given the spacecraft and planetary orbits). To do so, we constructed a model light curve by building a series of two-dimensional synthetic transit images (e.g., see Fig. 4). A circular subaperture (representing the disk of Venus) geometrically "occulted" portions of the model solar disk as the planet transited the photosphere. The model solar image was built, parametrically representing the center-normalized radially limb-darkened surface profile, $\mathrm{SB}(r)$, with a simple twoparameter form, $\mathrm{SB}(r)=1-a\left\{1-\left[\left(1-r^{2}\right)^{1 / 2}\right]^{b}\right\}$, where $r$ is the fractional solar radius, as suggested by Hestroffer \& Magnan (1998). Alternative limb-darkening formulations may be considered for planetary transit light-curve fitting (e.g., see Wittenmyer et al. [2005] in a recent improved solution, with stellar limb darkening, to the HD 20945b system parameters combining both photometric transit and radial velocity observations).

\subsection{Light-Curve Asymmetries}

As a result of the reflective spacecraft parallactic motion of Venus, the planet's apparent path across the Sun "nods" in

\footnotetext{
${ }^{6}$ Greg Kopp's TSI page is available at http://spot.colorado.edu/ koppg/TSI/.

7 See SORCE News at http://www.gsfc.nasa.gov/news-release/releases/2004/ h04-235.htm.
} 


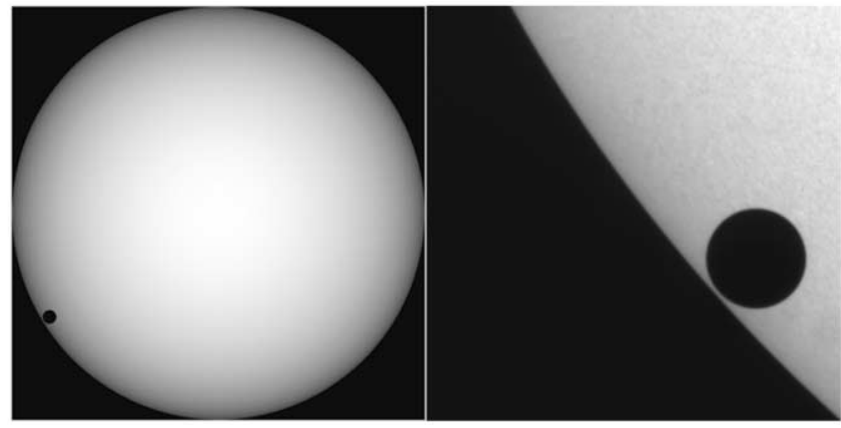

FIG. 4.-Full-disk synthetic transit image for 05:40 UT from a "best-fit" solution to the light-curve model (left), compared to a subarray extract from a TRACE WL image in the region of Venus and the solar limb (right).

heliocentric radius $(r)$, as illustrated in Figure 2. This motion induces asymmetries in the ACRIM radiometric light curve as Venus occults portions of the solar disk of differing surface brightnesses (flux densities) in a radially dependent manner due to solar limb darkening. One can see that during ingress Venus crosses from $r=1.0$ to 0.9 (where the limb darkening function has a very steep gradient; see Fig. 5) twice as slowly as it does from $r=0.9$ to 1.0 on egress. Hence, the downward slope of the ingress light curve is more shallow than during egress. In addition, small amplitude, orbit periodic variations in the TSI measured by ACRIM are expected as Venus oscillates between the brighter (smaller $r$ ) portion of the photosphere and positions closer to the solar limb (larger $r$ ). This is, at least in part, the cause of some of the "wiggles" that are seen at the bottom of the light curve. Aperiodic variations may result from intrinsic changes in the TSI over the same time interval or may arise as Venus occults isolated regions of the photosphere differing in local surface brightness (e.g., sunspots or smaller spatial scale localized features). Inspection of contemporaneous high-resolution broadband imagery from the TRACE spacecraft suggests that both effects are seen during the course of the transit. We later consider their statistical significance in the ACRIM 3 data set and how well the orbit periodic effect is separable from intrinsic variations in the global TSI over the same time interval, and also as Venus occults photospheric regions that may be intrinsically brighter or dimmer, in the context of TRACE imagery.

\subsection{Limb Darkening and Spacecraft Orbit Parallax}

A statistically significant shallow diminution in the radiometric flux density is seen after second contact but before mid-transit, i.e., approximately $-0.04 \%$ at $05: 50 \mathrm{UT}$ and approximately $-0.08 \%$ at 06:15 UT compared to approximately $-0.10 \%$ at mid transit, and a corresponding gradual rise before the loss of data due to Earth occultation on egress. This effect is fully attributable to radially differentiated solar limb darkening, with a strong photospheric radial surface brightness gradient as the limb of the Sun is approached. At 05:50 UT the center of Venus was $\sim 0.933 R_{\odot}$ from the heliocenter, whereas at mid-transit $(\sim 08: 35 \mathrm{UT})$ the center of Venus was $0.650 R_{\odot}$ from the heliocenter. This nonlinearity in impact distance with time arises, primarily, from the modulation in Venus's heliocentric velocity vector with respect to the limb (i.e., affecting the "limb crossing angles") induced by $A C R I M S A T$ 's orbital parallax.

\section{LIGHT-CURVE FITTING}

Model light curves were fitted to the observed TSI data via iterative, damped, nonlinear least-squares differential corrections

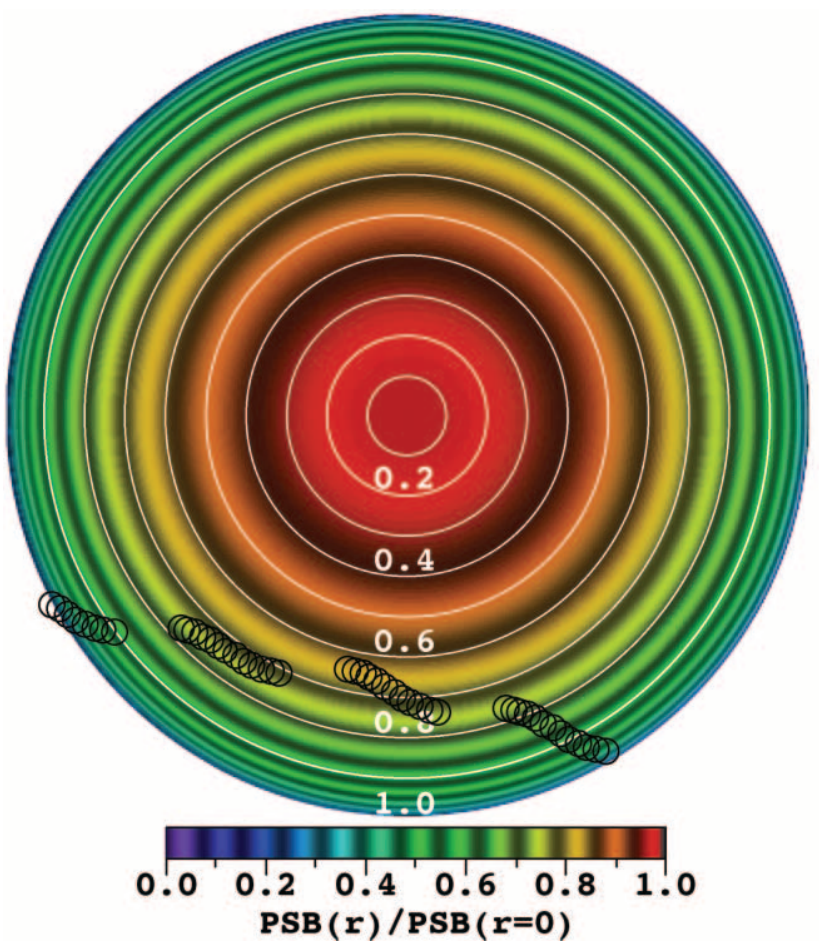

FIG. 5.-Center-normalized contours of photospheric surface brightness due to limb darkening characterized as described in $\S 5$ using $a=0.85, b=0.80$ as discussed in $\S 6$. White concentric circles indicate fractional distance from the heliocenter in increments of $0.1 R_{\odot}$. Small black circles indicate locations of Venus (drawn to scale) in 5 minute intervals while visible to ACRIMSAT.

(e.g., Schneider 1985), analogous to the method used by Schultz et al. (2004) in solving for the systemic parameters of the HD 209458A/B system using Hubble Space Telescope Fine Guidance Sensor transit light curves (with some differences in detail noted in $\S 5$ ). Geometrically, however, extrasolar planetary systems (such as HD 209458A/B) are effectively "at infinity" as seen from the Earth, so the ratio of planet : star angular and physical diameters are identical. This is not the case for the Venus transit geometry because of the close proximity of both objects to the Earth. Because of this, and the added nonlinearity in transit geometry due to the spacecraft orbit-induced planetary parallax, rather than adopting a parametric representation such as discussed by Schultz et al. (2004; which includes the time of midtransit, ratio of planet to stellar radii, and orbital inclination), we fitted the geometric times of transit contacts (CI-CIV), the transit depth (time-dependent decrement in TSI), and the solar limb darkening coefficients $a$ and $b$ as free parameters (each dependent on impact distance $r / R_{\odot}$ ). The time differentials CII-CI (duration of ingress) and CIV-CIII (duration of egress) were not constrained to be equal, as asymmetry in the observed light curve was expected due to the ACRIMSAT orbit-periodic parallax effects previously discussed. The light-curve model parameters that best fit the observations along with their formal $1 \sigma$ errors (after iterative convergence) are given in Table 1 ("measured").

\subsection{Transit Timing and Error Estimation}

Unlike a true extrasolar planetary transit, the measured times of contacts, and thus ingress, egress, and transit durations, are readily verifiable from our a priori knowledge of the Venus's (and ACRIMSAT's) orbits. The derived UT values of the egress contacts from our light-curve fitting, particularly CIV, are less well determined than for ingress. This is primarily due to the data 
TABLE 1

Light-Curve Solutions

\begin{tabular}{|c|c|c|c|}
\hline \multicolumn{4}{|c|}{ UT Contacts } \\
\hline & Measured & Predicted & \\
\hline CI........................... & $05: 12.95 \pm 3.1$ & $05: 10.32$ & $\ldots$ \\
\hline CII ………….... & $05: 32.42 \pm 3.8$ & $05: 35.82$ & $\ldots$ \\
\hline 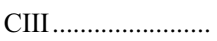 & $10: 55.57 \pm 4.4$ & $10: 59.25$ & $\ldots$ \\
\hline 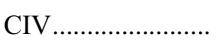 & $11: 32.92 \pm 5.2$ & $11: 29.50$ & $\ldots$ \\
\hline
\end{tabular}

\begin{tabular}{|c|c|c|c|}
\hline \multicolumn{4}{|c|}{ Durations } \\
\hline & Measured (min) & Predicted (min) & $\%$ Error \\
\hline Ingress ..................... & $19.47 \pm 4.9$ & 25.50 & -23.6 \\
\hline 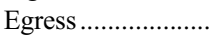 & $37.35 \pm 6.8$ & 30.25 & +23.5 \\
\hline 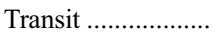 & $351.56 \pm 8.4$ & 351.31 & +0.07 \\
\hline \multicolumn{4}{|c|}{ Limb Darkening } \\
\hline а. & $0.85 \pm 0.01$ & $\ldots$ & $\ldots$ \\
\hline$b$ & $0.80 \pm 0.01$ & $\ldots$ & $\ldots$ \\
\hline \multicolumn{4}{|c|}{ Transit Depth (\%) } \\
\hline & $0.110 \pm 0.0002$ & $\ldots$ & $\ldots$ \\
\hline
\end{tabular}

loss from Earth occultation during CIII-CIV, but also because Venus's angular velocity component in the direction perpendicular to the solar limb was larger at egress than ingress (see Fig. 2). Nonetheless, in all cases the contact times determined from the TSI measures are consistent (within their $1 \sigma$ fitting uncertainties) with the back-predicted UT values from the definitive ACRIMSAT orbital ephemeris.

The measured durations of ingress (CII-CI) and egress (CIVCIII) are both in error (but in opposite sense) by about $23.5 \%$ from the true durations, which correspond to equivalent fractional errors in estimations of the planetary radius. If averaged this would reduce to a near zero error, but this is a coincidence of small sample statistics. A simple estimation of the expected uncertainty, in the absence of (in this case nonexisting) multiepoch observations, is predicated on combining the measurement uncertainties in quadrature, i.e., $\pm 22.0 \%(1 \sigma)$. This is in very good agreement with the percentage errors found in comparing the observationally determined, and ephemeris-predicted, contact times.

The full duration of the transit, measured as the time differential between the midpoints of ingress and egress, is formally uncertain by $2.4 \%$. This corresponds to an expectation of uncertainty in the determination of the ratio of the planetary to stellar radii. The much smaller percentage error in the transit duration determination from the TSI light-curve fitting is, again, just a happenstance of the nearly compensating measurement errors at ingress and egress.

From these data we would expect similar uncertainties in photometrically derived systemic parameters from extrasolar planetary transits - if sampled equivalently and measured with equal precision. Improvements would be expected with multiepoch (repeated) observations and with higher signal-to-noise ratio measurements for equivalent per sample integration times. In the absence of instrumental systematics, stellar variability and nonradial photospheric surface brightness (PSB) variations may ultimately limit transit detection thresholds for ETPs and the uncertainties in derived systemic parameters for ETPs above those thresholds.

\subsection{Intrinsic (Astrophysical) "Noise"}

We used the as-measured TSI variations in the flanking out-oftransit radiometry to assess "how well" (or deficient) our model light curve fits the data, i.e., how much of the fit residuals are due to instrumental measurement errors and intrinsic solar variations compared to imperfections in the model itself. "Variations" here not only include temporal variations in (area integrated) TSI but also spatial variations as Venus covers different parts of the photosphere that are not isotropic in intensity on small spatial scales.

In Figure 6 we show the difference in the dispersion in in-transit compared to pre-/post-transit model light-curve fit residuals, which is $+25 \%$ in "as measured" TSI variability (after subtracting out the model light curve). This in-transit increase in the dispersion in TSI of $0.002 \%$ during the transit is an order of magnitude larger than the dispersions about the median as-measured TSI's before and after the transit. One may posit one or more (1) instrumental, (2) systematic, and/or (3) and (4) real physical effects contributing to this increase as delineated below.

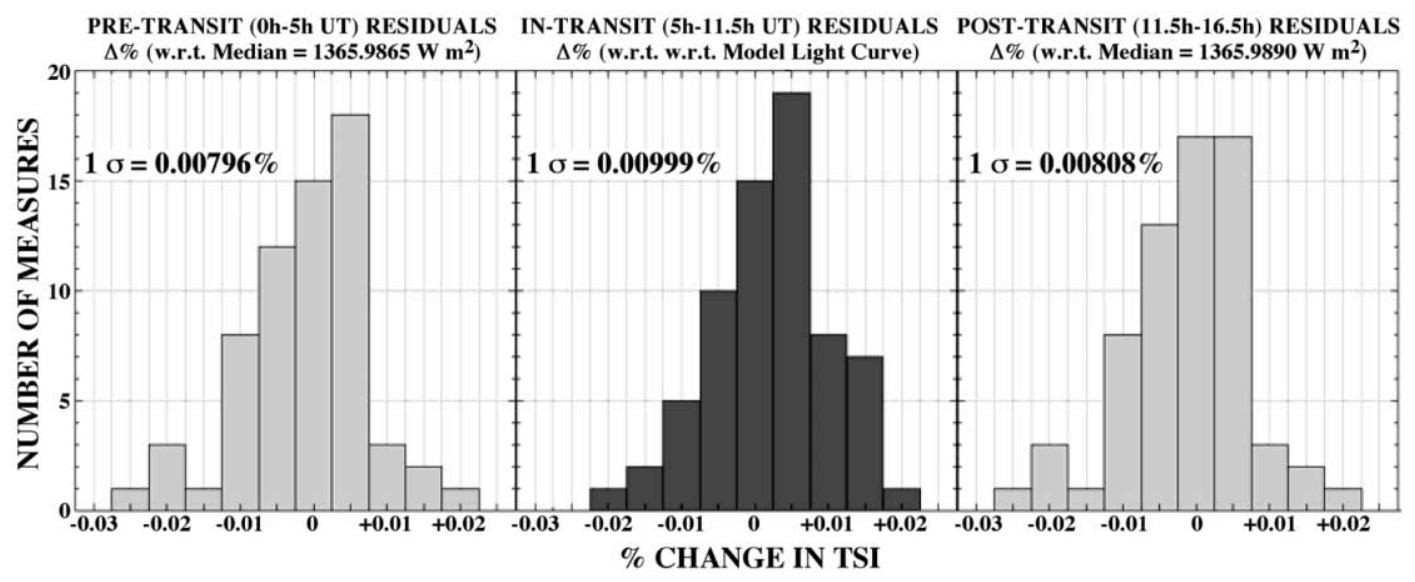

FIG. 6. - Left and right: Light-curve residuals from three contiguous orbits of pre- and post-transit data (each $\sim 5 \mathrm{hr}$ in duration) immediately before CI and after CIV, respectively. The median TSI measured for those two periods differed by only $0.0002 \%$, i.e., is "constant" within the measurement errors. The $1 \sigma$ variation in TSI from all (unbinned) $132 \mathrm{~s}$ "shutter cycle readouts" during each of these $5 \mathrm{hr}$ flanking periods is $\sim 0.008 \%$ of their respectively measured median TSI. The residuals from the light-curve fit during the intervening transit (of the same duration; middle) are $\sim 0.01 \%$ of the observed-minus-modeled TSI at the $1 \sigma$ level (an increase of $+25 \%$ over the pre- and post-transit periods). The apparent increase could arise either from (1) intrinsic (temporal) solar variations during the transit, (2) systematic deficiencies in the light-curve model as discussed in $\S 6.2$, or (3) shadowing by Venus of regions of differing photospheric surface brightness after removing the radial component due to limb darkening (§ 7). 
1. Uncertainties in the end-to-end wavelength-dependent system responsivity function for ACRIM under its very broad passband (i.e., its spectral sensitivity). These uncertainties are likely insignificant based on the ACRIM 3 cavity design and prelaunch testing.

2. Insufficient fidelity in the limb-darkening model. A quadratic model may be better, and could be tested, but a higher order (multiparametric) model is likely unjustified given the interrupted phase coverage and single-epoch-only nature of the light curve.

3. The effects of the atmosphere of Venus itself (absorption, aerosol scattering, and refraction).

4. The effect of Venus occulting regions of the photosphere differing in brightness on small spatial scales.

Neglecting or better characterizing (1), a higher order limbdarkening model (2) could be considered and tested by parametric variation bounded by the instrumental spectral sensitivity calibration. With that, rigorous detection limits for the planetary atmosphere (3) might be ascertained, within the uncertainties in the local variations in photospheric surface brightness on spatial scales of the diameter of Venus (4).

\section{A SURROGATE TO AN ETP TRANSIT}

The solar photosphere is nonisotropic in its surface brightness distribution on spatial scales both smaller, and larger, than the apparent diameter of a Venus. Hence, variations in measured TSI will arise during the transit that depend on the apparent planet/ solar crossing geometry. ACRIM 3, however, sees the Sun as a spatially unresolved source, so spatial (transit geometry dependent) and temporal variations in TSI cannot be decoupled from the radiometric data alone. This will also be the case for photometric transit light curves of ETPs (such as those to be obtained by the Kepler mission). In the test case of the transit of Venus, as a surrogate to an ETP transit, that degeneracy can be broken with contemporaneous high-resolution imaging of the solar photosphere.

At the time of the transit, between contacts II and III, the planetary disk of Venus occulted $0.0942 \%$ of the solar photosphere. But with an optically thick atmosphere extending to $\sim 60 \mathrm{~km}$ above the Cytherian surface (very high opacity up to the mesospheric cloud layer), the areal coverage was $0.0961 \%$. Thus, Venus's atmosphere effectively blocked an additional $0.002 \%$ of the received TSI (if not preferentially forward-scattered, re-

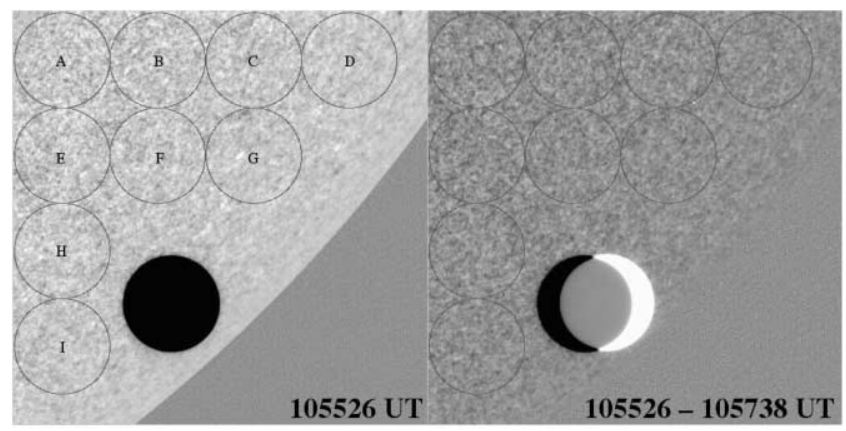

FIG. 7.-Left: Representative TRACE WL image, after removal of radial limb darkening, of Venus transiting the solar photosphere (one of 98 time-sliced images for this spacecraft pointing spanning 40 minutes of time). Photometric apertures (each enclosing 10,923 TRACE pixels) used to evaluate the temporal and spatial variability of the PSB on the size scale of Venus seen in projection are overlaid. Right: Difference image illustrating the change in PSB and the apparent movement of Venus over the $\sim 131 \mathrm{~s}$ interval of an ACRIM 3 shutter cycle at the indicated UT values. Both images are linear displays spanning the same dynamic display range (with the sky renormalized to midrange of the display stretches). Left: +2200 to $+3200 \mathrm{ADU}_{\text {pixel }}{ }^{-1}$. Right: -500 to $+500 \mathrm{ADU}_{\text {pixel }}{ }^{-1}$.

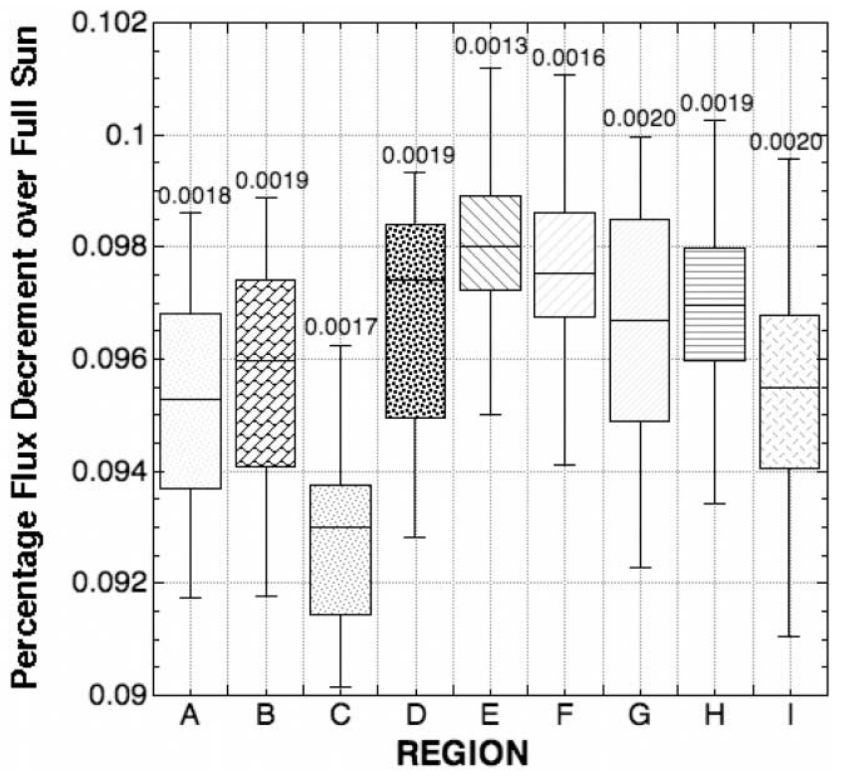

FIG. 8.-Variations in total solar flux density decrement ( $\triangle \%$ in the TRACE $0.1-1.0 \mu \mathrm{m}$ passband) due to photospheric occultation by a Venus-sized planet arising from temporal and spatial PSB variations. We illustrated the variations in photometric statistics for nine representative regions on the Sun in the vicinity of Venus during the time of the transit (corresponding to the positions designated A-I in Fig. 7) that would result if geometrically occulted. The height of rectangular boxes and vertical bars indicates the temporal variations in flux decrement that would result with repeated measures made (with the planetary occulter at the same position) over a 40 minute period of time. The box halfheights indicate upper and lower quartiles about measured medians (black lines in boxes) of photometric measures from 98 samples. The vertical bars indicate $\pm 2 \sigma$ variations about sample means, with $1 \sigma$ dispersions (in $\Delta \%$ ) annotated above each box. Variations arising from planetary occultations of the different regions resulting from anisotropies in the solar PSB may be assessed by comparing the interregion occultation statistics.

fracted, or reradiated by the atmosphere). We tested the ability to discriminate against a $1 \%$ equivalent increment in an Earthlike planetary radius (by the presence of Venus's opaque atmosphere) in light of both spatial and temporal solar photospheric "surface" brightness variations.

The solar PSB decreases radially from the heliocenter because of limb darkening. The PSB is also instantaneously nonheterogeneous on angular scales of $\sim 1^{\prime \prime}$ due to solar granulation, and on larger scales due to features such as sunspots. Thus, the TSI received at $A C R I M S A T$ (and corrected to $1 \mathrm{AU}$ ) is expected to vary as Venus occults different portions of the photosphere during its transit due to spatial variations in PSB, separate from also expected temporal variations.

\subsection{Photospheric Surface Brightness Variations}

We investigated the likely amplitudes of PSB variations after compensating for limb darkening that may affect ACRIM 3 measures of TSI with contemporaneous high-resolution imagery obtained with the TRACE spacecraft in its very spectrally broad WL channel $(\sim 0.1-1.0 \mu \mathrm{m})$.

We performed both temporally and spatially resolved limbdarkening-corrected differential photometry of regions flanking the location of Venus as it transited the photosphere (e.g., Fig. 7). With that we obtained statistical expectations of the levels of variability in TSI due to partial photospheric occultation at the angular scale of Venus (Fig. 8).

1. Temporal changes in TSI due to Venus occultation of any fixed region of the Sun tested (e.g., denoted A-I in Fig. 7) were 
found to be $\pm 0.0018 \%$ at the $1 \sigma$ level (compared to a $0.0019 \%$ expected change in signal) with inter-region variations in internal dispersions of $\pm 0.00022 \%$. Hence, a sensitivity to the presence versus the absence of a Venus-like opaque planetary atmosphere was tested at only a $1.05 \sigma$ level of confidence.

2. TSI variations due to spatial anisotropies in PSB on Venussized angular scales were found dispersed by $\pm 0.0015 \%$ at the $1 \sigma$ level about an expected decrement in TSI of $0.0961 \%$ due to the presence of Venus imposed on the photosphere with compensation for limb darkening (i.e., a $1.3 \sigma$ "detection" of the atmosphere of Venus).

The virtual equivalence in amplitude of the spatial and temporal variations implicates no significant systematic effects in this data set from large spatial scale PSB variations (after proper limbdarkening compensation) in excess of limiting detection sensitivities from temporal effects. We note from full-disk TRACE (and supplemental ground-based) imagery that, serendipitously, there were no large-scale photospheric structures (i.e., sunspots) that might otherwise have differentially affected the area-integrated solar brightness due to occultation by Venus. The differential effects of sunspot (or starspot) transits, however, is anticipated and would additionally complicate transit light-curve analysis.

\section{SUMMARY}

The ACRIM 3 radiometric observation of the $5.5 \mathrm{hr}$ duration ToV, with single 2.2 minute readout measures precise to one part in $10^{-4}$ (see Fig. 3), clearly demonstrates, by analogy, the ability to detect ETP stellar transits photometrically with scalably comparable instrumental sensitivities. By comparison, the goal for Kepler differential photometry, predicated on its expected $1 \sigma$ noise performance estimation of $\sim 2 \times 10^{-5}$ for an $m_{V}=12$ solarlike star, including photon shot noise and stellar variability, will yield $4 \sigma$ terrestrial planet detections for a single $2-16 \mathrm{hr}$ transit ${ }^{8}$ (Koch et al. 1998).

\footnotetext{
8 ACRIM 3's temporal sampling of the ToV was compromised by interruptions due to Earth occultations. Photometric sampling by Kepler, which is to be placed in a solar orbit, will not be so affected.
}

The amplitudes and dispersions of both the temporal and spatial PSB variations of Sun-like stars would preclude discriminating with sufficient statistical significance the presence versus absence of a Venus-like opaque planetary atmosphere for an Earth-sized transiting planet by transit observations of solarlike stars with ACRIM 3-like sampling (even if uninterrupted). Hence, following ETP detection (and orbital characterization with multiepoch photometric observations), alternate strategies, such as spectroscopic capabilities on subsequent missions (e.g., an integral field spectrograph on $T P F-C$ ), must be considered to definitively move from detection to characterization of extrasolar terrestrial planets.

The spatially unresolved Venus transit light curve obtained by $A C R I M S A T$ (and a similar one obtained by SORCE/TIM) is the closest proxy to an ETP transit that exists. Given our a priori knowledge of the star/planet system geometry and properties, this unique data set may be exploited to investigate the detectability of ETP transit observation methods contemplated by future space-based terrestrial planet-finding missions. With sufficient photometric precision, proper characterization of the effects of stellar limb darkening may yield information on the vertical structures in stellar atmospheres and have the potential of revealing the existence of more distended planetary atmospheres of planets transiting more quiescent stars.

We gratefully acknowledge the cooperation and assistance of Karel Schrijver, Ted Tarbell, and other members of the TRACE and ACRIM science, planning, and operations teams in helping us to secure the high-resolution imagery and time-resolved radiometric data used in this investigation. G. S. and J. M. P. thank the Committee for Research and Exploration of the National Geographic Society for their support of our Venus transit studies. J. M. P.'s work is supported in part by grants from NASA's planetary and solar-terrestrial programs and the Rob Spring Fund at Williams College. The National Aeronautics and Space Administration provides support for R. C. W. at Columbia University under contract NNG004HZ42C.
Alonso, R, et al. 2004, ApJ, 613, L153

Beichman, C. A. 1998, Proc. SPIE, 3350, 719

Borucki, W. J., et al. 2003, in ASP Conf. Ser. 294, Scientific Frontiers in Research on Extrasolar Planets, ed. D. Deming \& S. Seager (San Francisco: ASP), 427

Charbonneau, D., Brown, T. M., Latham, D. W., \& Mayor, M. 2000, ApJ, 529, L45

Charbonneau, D., Brown, T. M., Noyes, R. W., \& Gilliland, R. W. 2002, ApJ, 568,377

Hestroffer, D., \& Magnan, C. 1998, A\&A, 333, 338

Koch, D. G., Borucki, W., Webster, L., Dunham, E., Jenkins, J., Marriott, J., \& Reitsema, H. J. 1998, Proc. SPIE, 3356, 599

Macintosh, B., et al. 2001, in ASP Conf. Ser. 244, Young Stars Near Earth: Progress and Prospects, ed. R. Jayawardhana \& T. Greene (San Francisco: ASP), 309

\section{REFERENCES}

Mayor, M., \& Queloz, D. 1995, Nature, 378, 355

Pasachoff, J. M., Schneider, G., \& Golub, L. 2005, in IAU Colloq. 196, Transits of Venus: New Views of the Solar System and Galaxy, ed. D. W. Kurtz (Cambridge: Cambridge Univ. Press), 242

Schneider, G. 1985, Ph.D. thesis, Univ. Florida

Schneider, G., Pasachoff, J. M., \& Golub, L. 2004, Icarus, 168, 249

Schneider, G., \& Silverstone, M. 2003, Proc. SPIE, 4860, 1

Schultz, A. B., et al. 2004, in AIP Conf. Proc. 713, The Search for Other Worlds: Fourteenth Astrophysics Conference, ed. S. S. Holt \& D. Deming (New York: AIP), 161

Strong, K. T., Bruner, M., Tarbell, T., Title, A., \& Wolfson, C. J. 1994, Space Sci. Rev., 70, 119

Willson, R. C., \& Mordvinov, A. V. 2003, Geophys. Res. Lett., 30, 1199

Wittenmyer, R. A., et al. 2005, ApJ, 632, 1157 